\title{
12W UV laser based on Air-cooled Double-end-pumped Intra-cavity
}

\author{
Zhou Yuchao, a , Zhao shengyu ${ }^{1, b}$, Zhou Yongquan ${ }^{2, c}$ and Song Yuli, ${ }^{1, d}$ \\ ${ }^{1}$ Shenzhen Hymson Laser Technologies Co., Ltd, , Shenzhen, GuangDong, China \\ ${ }^{2}$ Shenzhen Institute of Information Technology, Shenzhen, GuangDong, China \\ apeter@hymson.com, bzhaosy@hymson.com, czhouyq@sziit.edu.cn, csongyuli@hymson.com
}

Keywords: Double-end-pumped; UV laser; Triple frequency; Micro-channel, Heatsink

Abstract. 12W UV laser generator is developed as a second harmonic generator and a third harmonic generator, in which a folded resonance cavity is composed of two laser diode side-pumped Nd:YAG modules, an electro-optic Q-switch, two LBO crystals. The air-cooled double-end-pumped Intra-cavity is studied and designed, the laser wavelength is $355 \mathrm{~nm}$, its maximum output power is $12.34 \mathrm{~W}$ at repetition rate of $15 \mathrm{kHz}$. The corresponding pulse width is $12 \mathrm{~ns}$. And the optical-optical efficiency of the fundamental laser is about $41 \%$.

\section{Introduction}

UV laser is widely being used in fine and precise laser machining[1-2]. Currently, the output power of UV laser generator, whose cavity is cooled by air, is not over $10 \mathrm{~W}$ due to the poor thermal dissipation of laser cavity, so water-cooled system is needed in the laser cavity of high power[3].

In the pursuit of higher power, the cost and volume of UV laser equipment have to increase. The main approach to make bigger output power is to increase the number of fundamental frequency modules[4]. UV and green laser are obtained through frequency doubling of high power output of fundamental frequency laser, which are usually used for fine processing market. With the increase of the power, the requirements of the stability of the UV and green laser equipment have become higher and more stringent[5]. Lower power consumption, maintenance-free and smaller size ones are in great demand. Thus, it is an inevitable trend that water-cooled laser equipments are replaced by air-cooled ones due to the excellent performance of stability of air-cooled UV laser.

In this paper, a system of double-end-pumping is applied to get high power solid-state $355 \mathrm{~nm} \mathrm{UV}$ laser. Two semiconductor laser diodes (LD) are used as pumping sources to end-pump the laser crystal. $808 \mathrm{~nm}$ from LD is coupled into the crystal system through aspherical lens to produce $1064 \mathrm{~nm}$ laser, which oscillates in the resonator composed by five flat mirrors and is modulated by Q switch. $1064 \mathrm{~nm}$ laser goes through the frequency-doubling crystal to transfer into 532nm. The remaining $1064 \mathrm{~nm}$ and newly generated $532 \mathrm{~nm}$ interact in the frequency-tripling crystal to get output of $355 \mathrm{~nm}$ UV laser. In the meantime, TEC chips are needed to control the temperature of frequency-doubling crystal and frequency-tripling crystal respectively ,but itself heat of each TEC chip should be dissipated, a heatsink structure with micro-channel for effectively air-cooling is developed.

\section{Double-end-pumped Intra-cavity}

Figure 1 shows there are two same semiconductor laser diodes (LD1 and LD2) to play the role of pumping sources to end-pump the laser crystal, each diode output power is $40 \mathrm{~W}$. $808 \mathrm{~nm}$ laser from LD is coupled into the crystal system through aspherical lens to produce $1064 \mathrm{~nm}$ laser, which oscillates in the resonator composed by five flat mirrors (M1, M2, M3, M4 and M5) and is modulated by Q switch. $1064 \mathrm{~nm}$ laser goes through the frequency-doubling crystal $\mathrm{S}$ to transfer into $532 \mathrm{~nm}$. The remaining $1064 \mathrm{~nm}$ and newly generated $532 \mathrm{~nm}$ interact in the frequency-tripling crystal T to get output of $355 \mathrm{~nm}$ UV laser. A TEC cooling chip is placed above the frequency-doubling crystal S and frequency-tripling crystal $\mathrm{T}$. This design makes the laser cavity have a larger mode volume and a smaller spot at the same time to improve the conversion efficiency. 
Compared to the external cavity frequency doubling, intracavity frequency doubling has the following advantages: the light with relatively high intensity in the cavity can achieve nonlinear frequency conversion. It is not necessary for beam diameter through crystals to be focused to a very small spot, which will greatly reduce the chances of damaging the crystal. Moreover, the requirement of coating on the crystal is not so sophisticated in this method. And laser can have longer working life.

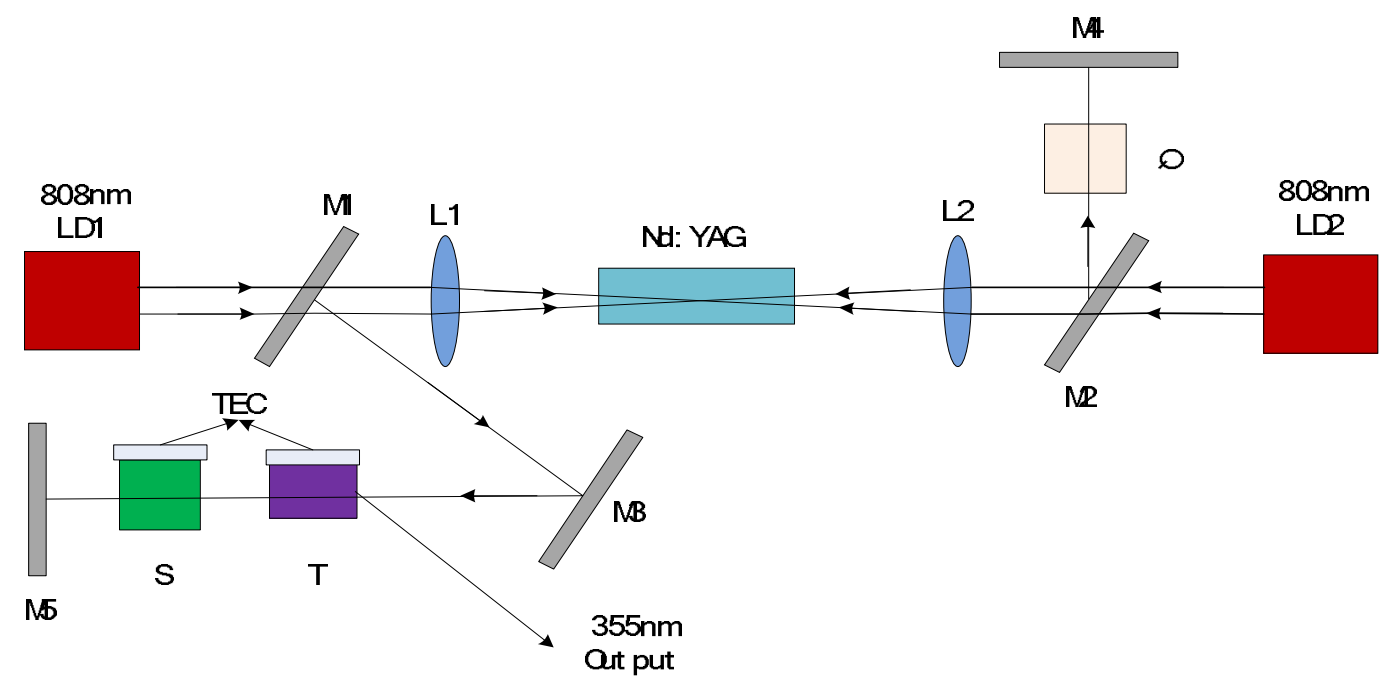

Fig.1 Double-end-pumped Intra-cavity cooled by air

The current air-cooled end-pumped lasers can achieve a stable output of the infrared laser, but stability of the output power is reduced after adding multiplier in the same structure. There are two main reasons: Firstly, the optical cavity's requirement of the mechanical precision of frequency-doubled laser is higher than that of infrared lasers and the air-cooled heat sink design will affect the deformation of the cavity; Secondly, the efficiency of frequency-doubling is related with the multiplier crystal's temperature. In spite of the use of high-precision chilling plates in the multiplier module, the heat source in the optical cavity will heat the whole optical cavity, thereby changing the temperature around the multiplier module, reducing the conversion efficiency of the multiplier module, leading to the power instability of frequency-doubling laser.

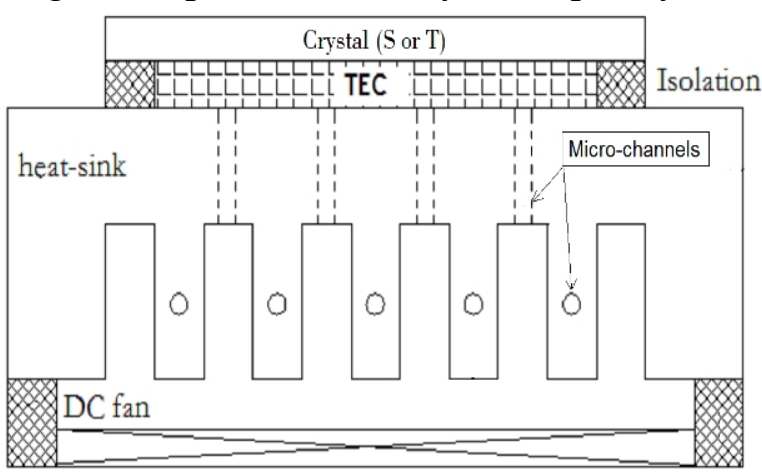

Fig. 2 Heatsink structure with micro-channels

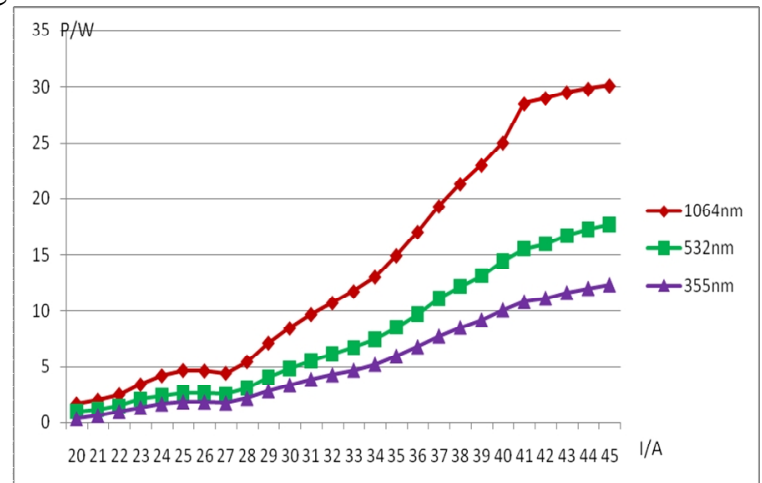

Fig. 3 Output power at diffrent wavelength

A TEC cooling chip plays a key role of temperature control of frequency-doubling crystal $\mathrm{S}$ and frequency-tripling crystal $\mathrm{T}$ in our reasech. Those crystals are installed on a heatsink, which is used to cool TEC chips by air. As a commom practice, the thickness of ribs could not be reduced too much and the number of ribs could be increased too much due to the restriction of extrusion technology of heatsink, it means the bottleneck of heat dissipation of traditional heatsink exists. In order to overcome the bottleneck, an innovated heatsink structure with micro-channnels are developed as show in Fig.2. The diameter of micro-channel is from $0.2 \mathrm{~mm}$ to $0.5 \mathrm{~mm}$ depending on the thickness of ribs of heatsink. Although micro-channnels increase the manufacturing cost of laser cavity, but those do increase the heat disspating area and improve the efficiency of heat dissipation consequently. 


\section{Results and discussion}

To assess the optical conversion efficiency of the laser, the fundamental frequency of $1064 \mathrm{~nm}$, frequency-douled 532nm and frequency-tripled 355nm laser output power are measured respectively at Q-switched repetition rate of $15 \mathrm{kHz}$. Firstly, 355nm UV laser is obtained in the setup shown in Fig.1. Then remove the frequency-tripling crystal T, 532nm green laser can be measured. Lastly, remove the parts from M3 to M5, we can get fundamental frequency of 1064nm. The results are shown in Figure 3.

When the pump current reaches $45 \mathrm{~A}$, output power of $1064 \mathrm{~nm}, 532 \mathrm{~nm}$ and $355 \mathrm{~nm}$ light are $30.1 \mathrm{~W}$, $17.6 \mathrm{~W}$ and $12.34 \mathrm{~W}$ respectively at a repetition rate of $15 \mathrm{kHz}$. Therefore, the optical conversion efficiency of fundamental frequency is about $41 \%$. Optimized design of cavity structure and excellent phase matching ensures this high conversion efficiency.

Stable output of UV pulse at different repetition rates is obtained by adjusting the modulation frequency of Q-switch. Figure 4 and Figure 5 are laser output power and pulse width changing with pumping current, respectively, at the repetition rate from $10 \mathrm{kHz}$ to $50 \mathrm{kHz}$.

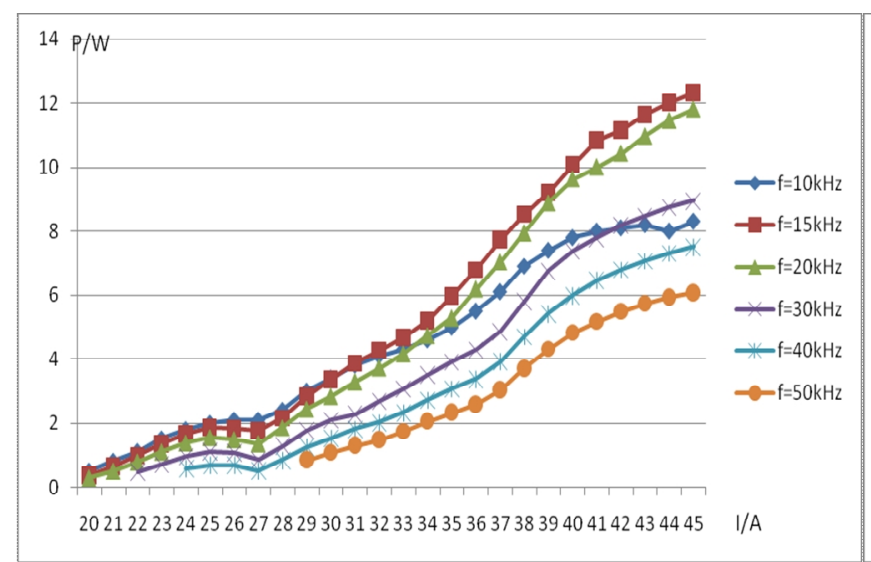

Fig.4 Output power of $355 \mathrm{~nm}$ at different repetition rates

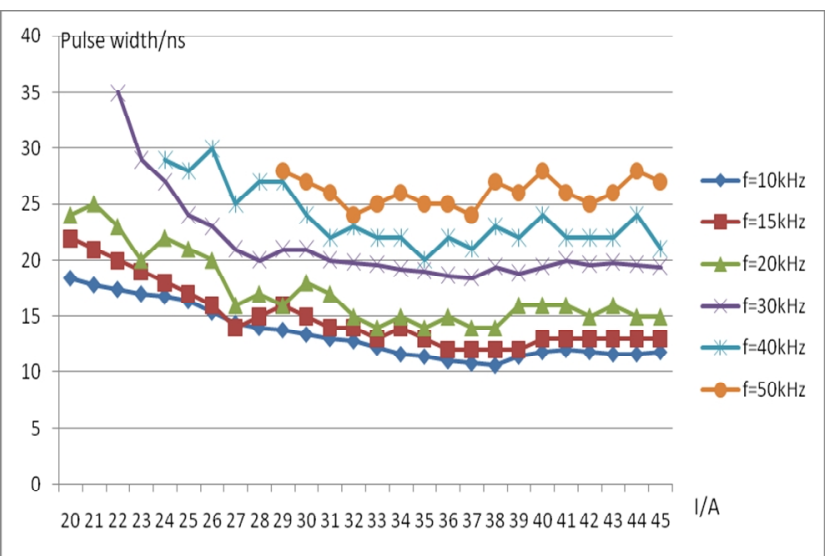

Fig.5 Pulse width of $355 \mathrm{~nm}$ at different repetition rates

At the same repetition rates, average output power increases with the increase of pump power and the maximum output is about $12.34 \mathrm{~W}$. When the pump current reaches a relatively high value, the output comes to a saturated trend. It can be explained that the beam quality of fundamental frequency light deteriorates at high-power pumping, resulting in decreased efficiency of frequency doubling. At the same pump power, the average output power reduces as the repetition rate increases in the range greater than $20 \mathrm{kHz}$. And the repetition frequency of $10 \mathrm{kHz}, 15 \mathrm{kHz}$ and $20 \mathrm{kHz}$ failed to be consistent with this relationship. It is directly related to the lifetime of the upper level of the laser working medium, which affects the population accumulated in the upper level during the Q switch off-time. When the interval period of the modulated pulse gets close to the lifetime of the crystal's upper level(the corresponding frequency of the crystal used in the experiment is about $15 \mathrm{kHz}$ ), the population accumulated in the upper level reaches the maximum during the period when Q-switch is off. When the modulation frequency is less than $15 \mathrm{kHz}$, the population inversion reduces due to the spontaneous emission. When above $15 \mathrm{kHz}$, the population accumulated in the upper level is only related to the modulated frequency of the Q-switch. As the frequency further increases, the interval period of Q-switch is shortened and the population accumulated on the upper level during the switch-off time reduces under a certain pump power. Therefore, the output power reduces as frequency increases in the range of above $20 \mathrm{kHz}$ while the relationship around $15 \mathrm{kHz}$ is not so straightforward.

The graph of the Q-switched pulse width changing with pump current is shown in Fig. 4. At the same repetition rate, average output power increases and pulse width decreases with the increase of the pump power. When the pump power is greater than the threshold value, the pulse width is relatively broad since the fluorescent effect. As the power increases, the net gain coefficient in the cavity keeps increasing, the number of photons and population inversion decrease more rapidly. The leading and trailing edges of pulses are both contracted so that the width reduces, eventually tending to a constant, 
i.e., the intracavitary photon lifetime. Figure 6 is a typical pulse shape displayed on the oscilloscope. The feature of the pulse waveform is having a steep leading edge and gentle trailing edge.

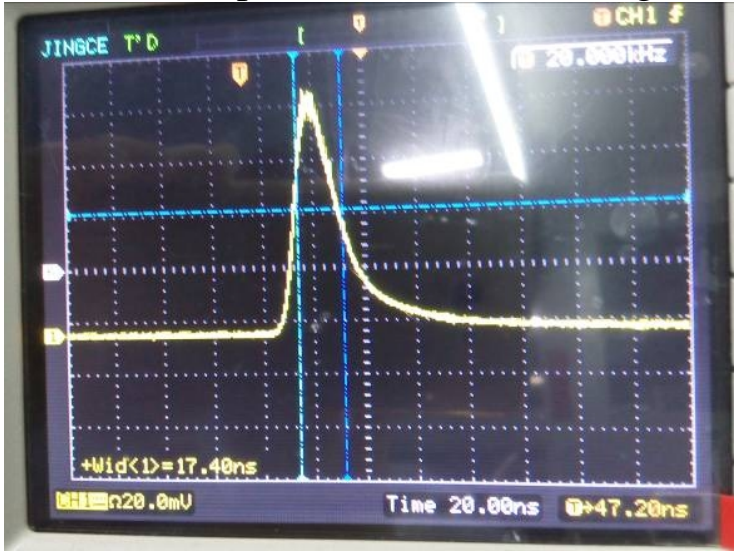

Fig.6 A typical single pulse

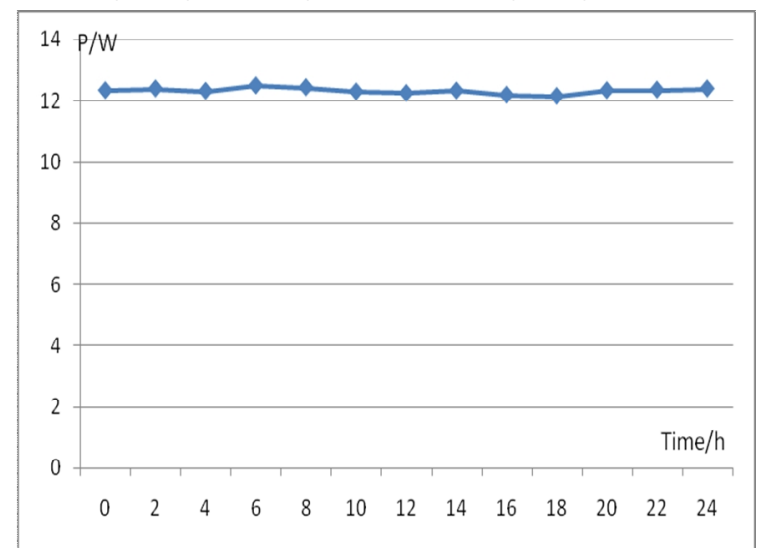

Fig.7 Output power in 24 hours

In order to assess the long-term stability of the laser, as shown in Figure 7, the test of 355nm laser output power stability is done at $12.3 \mathrm{~W}$ when continuously working for 24 hours. Test interval is $2 \mathrm{~h}$. Laser stability can be calculated to be $\pm 1.9 \%$.

\section{Conclusions}

A folded resonance cavity is composed of two laser diode side-pumped Nd:YAG modules, an electro-optic Q-switch, two LBO crystals separately as a second harmonic generator and a third harmonic generator. The relationships of output power and pulse width with the changing current are discussed at different repition rates. A maximum output power at $355 \mathrm{~nm}$ of $12.34 \mathrm{~W}$ is obtained at repetition rate of $15 \mathrm{kHz}$. The corresponding pulse width is $12 \mathrm{~ns}$. And the optical-optical efficiency of the fundamental laser is about $41 \%$. The stability of the laser output power is tested to be about $\pm 1.9 \%$ in 24-hour continuous operation.

\section{Acknowledgements}

This work was financially supported by the Shenzhen Science and Research Foundation (CXZZ2014 0416104115582), Shenzhen Science and Research Foundation (GJHZ20150316112419786), Guangdong Science and Research Foundation (2015A050502006).

\section{References}

[1] Andrew Dunn, JesperV.Carstensen, KrystianL.Wlodarczyk. 2014.Nanosecond laser texturing for high friction applications. Optics and Lasers in Engineering. 62, 9-16.

[2] Wu Yuwen, Guo Liang, Zhang Qingmao. 2012. Study on process of marking two-dimentional codes on air circuit breakers by UV laser. High Power Laser And Particle Beams. 24(6), $1329-1334$.

[3] Liu Qiang, Yan Xingpeng, Chen Hailong. 2010. New Progress In High-Power All-Solid_State Ultraviolet Laser. Chinese Journal of Lasers. 37(9). 2289-2298.

[4] S R I Gabran, R R Mansour, M M A Salama. Maskless pattern transfer using 355nm laser [J]. Optics and Lasers in Engineering, 2012, 50(5):710-716.

[5] CharlessX. Wang, Gary Y.Wang, AcleV.Hicks, David R.Dudley, H.Y.Pang,and N.Hodgson. High-power Q-switched TEM00 mode diode pumped solid state lasers with > 30W output power at 355nm [J]. Proc.SPIE,2006,(6100):6100.1-6100.9. 